Proceedings of the Edinburgh Mathematical Society (2006) 49, 383-389 (C) DOI:10.1017/S0013091504001312 Printed in the United Kingdom

\title{
POSITIVE SOLUTIONS TO A NON-RADIAL SUPERCRITICAL KLEIN-GORDON-TYPE EQUATION
}

\author{
SEBASTIÁN LORCA ${ }^{1}$ AND MARCELO MONTENEGRO ${ }^{2}$ \\ ${ }^{1}$ Departamento de Matemática, Universidad de Tarapacá, \\ Casilla 7D, Arica, Chile (slorca@uta.cl) \\ ${ }^{2}$ Departamento de Matemática, Universidade Estadual de Campinas, \\ Instituto de Matemática, Estatística e Computação Científica, CP 6065, \\ CEP 13083-970, Campinas, SP, Brazil (msm@ime.unicamp.br)
}

(Received 9 November 2004)

\begin{abstract}
We find positive solutions to a nonlinear equation of Klein-Gordon type. Our analysis is carried out by truncating the related functional and estimating mountain pass solutions by Moser's iterative scheme.
\end{abstract}

Keywords: Klein-Gordon equation; supercritical; quasilinear; $m$-Laplacian; mountain pass

2000 Mathematics subject classification: Primary 35B25; 35B34; 35J20; 35J50; 35J60

\section{Introduction}

In this paper we deal with the equation

$$
-\Delta_{m} u+|u|^{m-2} u-a(x)\left(\lambda|u|^{p-1} u-|u|^{q-1} u\right)=0 \quad \text { in } \mathbb{R}^{N},
$$

where $N \geqslant 3,1<m<N, m-1<p<m^{*}-1 \leqslant q, m^{*}=m N /(N-m)$ and $\lambda$ is a positive parameter. We assume throughout this note that

$$
\begin{array}{cl}
0 \leqslant a(x)<K & \text { for } x \in \mathbb{R}^{N} \text { and } K>0 \text { a constant, } \\
a(x)>0 & \text { for } x \in B \subset \mathbb{R}^{N}, \text { where } B \text { is a ball }
\end{array}
$$

and

$$
\lim _{|x| \rightarrow \infty} a(x)=0 .
$$

A similar problem was treated in $[2]$ where $m=2$ and $a \equiv 1$ for the equation

$$
-\Delta u-g(u)=0 \quad \text { in } \mathbb{R}^{N} .
$$

The nonlinearity $g$ is subcritical, for instance, $g(u)=u^{p}-u^{q}-u$ with $1<p, q<$ $(N+2) /(N-2)$. The authors were able to handle more general nonlinearities $g(u)$ under suitable assumptions. 
Also, in [1] the authors solve (1.1) with $m=2, a \equiv 1,1<p<(N+2) /(N-2)$ and $q>p$. This supercritical problem is treated in radial coordinates by minimizing $\int \frac{1}{2}|\nabla u|^{2}$ subject to the constraint $\int G(u)=1$, where $G$ is the primitive of $g$ with $G(0)=0$. They recover compactness by working in $H_{\text {rad }}^{1}\left(\mathbb{R}^{N}\right)$ and using the Strauss lemma [7]. The Lagrange multiplier is ruled out by rescaling the equation, since $a \equiv 1$. Their result is sharp in the sense that they determine a constant $\lambda_{0}>0$ such that (1.1) has a positive (radial) solution if and only if $\lambda>\lambda_{0}$. Their approach also applies if one considers the $m$-Laplacian instead of the Laplacian operator. But if one incorporates a weight function $a(x)$ as in (1.1), even a radial one, then their techniques are not applicable, because there is no way to rescale the problem when one finds a constrained minimizer.

The functional setting to attack the supercritical problem (1.1) is by considering

$$
J: W^{1, m}\left(\mathbb{R}^{N}\right) \cap L^{1+q}\left(\mathbb{R}^{N}\right) \rightarrow \mathbb{R}
$$

defined by

$$
J(u)=\frac{1}{m} \int\left(|\nabla u|^{m}+|u|^{m}\right)-\int a(x)\left(\frac{\lambda}{p+1}|u|^{p+1}-\frac{1}{q+1}|u|^{q+1}\right) .
$$

For ease of notation, all integrals throughout the paper are computed in $\mathbb{R}^{N}$, unless otherwise mentioned. The functional $J$ has no clear geometry, for instance, it does not satisfy the 'mountain pass theorem' assumptions in $W^{1, m}\left(\mathbb{R}^{N}\right)$. Note that zero is not a local minimizer in the case $q>m^{*}-1$. And, even if $q=m^{*}-1$, there is a lack of compactness. Unlike $[\mathbf{1}, \mathbf{2}]$, we cannot work with radial functions, since $a(x)$ may be nonradial. We then truncate the nonlinearity $g(x, u)=a(x)\left(\lambda|u|^{p-1} u-|u|^{q-1} u\right)$ in order to make the problem appropriately subcritical. The principal part, $-\Delta_{m} u$, of the equation makes difficult the obtainment of estimates, since we cannot bootstrap using the classical $L^{p}$ theory involving linear elliptic operators. The new (truncated) functional $\hat{J}$ satisfies the mountain pass geometry. In this way, there is a Palais-Smale (PS) sequence. Since $a(x)$ decays to zero, this allows us to show that the sequence has non-trivial limit $u$, by [5]. $u$ is then a critical point of $\hat{J}$. To conclude that $u$ is indeed a solution of the original problem (1.1), we follow a Moser iterative scheme to find an $L^{\infty}$ bound for $u$ (see [6]). Actually, one concludes that $\|u\|_{L^{\infty}\left(\mathbb{R}^{N}\right)}$ is small for sufficiently large $\lambda$.

There are works related to ours where, say, non-autonomous subcritical problems are addressed. In [3] the authors found exponentially decaying solutions by a weighted-space approach to solving the equation. The equations considered in $[\mathbf{4}, \mathbf{8}]$ are more closely related to ours, except for the fact that, in their context, $a(x)$ does not tend to zero at infinity.

\section{Statements and proofs}

We begin with a non-existence result. Henceforth, we assume that (1.2)-(1.4) hold.

Theorem 2.1. There is a constant $\tilde{\lambda}>0$ such that, for $\lambda \leqslant \tilde{\lambda}$, there is no non-trivial solution. 
Proof of Theorem 2.1. Multiply Equation (1.1) by a possible positive solution $u$. Then

$$
\begin{aligned}
\int|\nabla u|^{m} & =\int a(x)\left(\lambda|u|^{p+1}-|u|^{q+1}\right)-|u|^{m} \\
& =\int_{u \leqslant \lambda^{1 /(q-p)}} a(x)\left(\lambda|u|^{p+1}-|u|^{q+1}\right) \\
& \quad-\int|u|^{m}+\int_{u>\lambda^{1 /(q-p)}} a(x)\left(\lambda|u|^{p+1}-|u|^{q+1}\right) \\
& \leqslant \int_{u \leqslant \lambda^{1 /(q-p)}} a(x)\left(\lambda|u|^{p+1}-|u|^{q+1}\right)-\int|u|^{m} \\
& \leqslant \int_{u \leqslant \lambda^{1 /(q-p)}} K\left(\lambda|u|^{p+1}-|u|^{q+1}\right)-|u|^{m} .
\end{aligned}
$$

Let $h(\lambda, s)=\lambda K s^{p+1}-K s^{q+1}-s^{m}$ (see (1.2)). A simple calculation shows that there exists a $\tilde{\lambda}$ such that, for $0 \leqslant \lambda \leqslant \tilde{\lambda}, h(\lambda, s) \leqslant 0$ for every $s \geqslant 0$. Consequently,

$$
\int|\nabla u|^{m} \leqslant \int_{u \leqslant \lambda^{1 /(q-p)}} h(\lambda, u) \mathrm{d} x \leqslant 0 .
$$

Hence, $u \equiv 0$ for $\lambda \leqslant \tilde{\lambda}$.

Remark 2.2. Note that the previous theorem is true for $1<m<\infty$.

The existence result reads as follows.

Theorem 2.3. There is a constant $\lambda^{*}>0$ such that for $\lambda>\lambda^{*}$ there is a weak solution $u>0$ belonging to $W^{1, m}\left(\mathbb{R}^{N}\right) \cap L^{\infty}\left(\mathbb{R}^{N}\right)$.

Proof of Theorem 2.3. Define

$$
f(\lambda, u)= \begin{cases}0 & \text { if } u \leqslant 0 \\ \left(1-\frac{u^{q-p}}{\lambda}\right) u^{p} & \text { if } 0<u \leqslant \varepsilon \\ \left(1-\frac{\varepsilon^{q-p}}{\lambda}\right) u^{p} & \text { if } u>\varepsilon .\end{cases}
$$

We also assume that $\lambda>1$, because this allows us to take $\varepsilon$ in the interval $(0,1)$ uniformly in $\lambda$. Here $\varepsilon$ is chosen in such a way that

$$
\left(1-\varepsilon^{q-p}\right) u^{p} \leqslant f(\lambda, u) \leqslant u^{p}
$$

and

$$
\theta F(\lambda, u) \leqslant f(\lambda, u) u \text { for } u>0 \text { for some constant } \theta>m,
$$

where $F$ is the primitive of $f$ with $F(\lambda, 0)=0$. We now study the modified equation

$$
-\Delta_{m} u+|u|^{m-2} u-\lambda a(x) f(\lambda, u)=0 .
$$


The corresponding functional $\hat{J}: W^{1, m}\left(\mathbb{R}^{N}\right) \rightarrow \mathbb{R}$ is defined by

$$
\hat{J}(u)=\frac{1}{m} \int|\nabla u|^{m}+|u|^{m}-\int \lambda a(x) F(\lambda, u) .
$$

The functional $\hat{J}$ satisfies the mountain pass geometry. Hence there is a PS sequence $u_{n} \in W^{1, m}\left(\mathbb{R}^{N}\right)$, that is, $\hat{J}\left(u_{n}\right) \rightarrow c$ and $\hat{J}^{\prime}\left(u_{n}\right) \rightarrow 0$. Our aim is to prove that a subsequence of $u_{n}$ converges to some $u$, thus finding a critical point of $\hat{J}$. We need also to verify that $u \not \equiv 0$.

By multiplying Equation (2.4) by $u_{n}$, using the fact that $\hat{J}\left(u_{n}\right) \rightarrow c$ and by (2.3), we can conclude that $u_{n} \rightarrow u$ in $W^{1, m}\left(\mathbb{R}^{N}\right)$. Since $\hat{J}^{\prime}\left(u_{n}\right) \rightarrow 0$, by $(2.2)$ one has

$$
\left\|u_{n}\right\|_{W^{1, m}\left(\mathbb{R}^{N}\right)}^{m} \leqslant C\left\|u_{n}\right\|_{L^{p+1}\left(\mathbb{R}^{N}\right)}^{p+1}+\delta_{n}\left\|u_{n}\right\|_{W^{1, m}\left(\mathbb{R}^{N}\right)},
$$

where $\delta_{n} \rightarrow 0$. By a variant of the result of [5], there is a sequence $x_{n}$ such that

$$
\int_{B_{1}\left(x_{n}\right)}\left|u_{n}\right|^{m}>\bar{c}>0
$$

for some constant $\bar{c}$. Passing to a subsequence if necessary, there are only two cases to analyse. If there is a constant $K>0$ such that $\left|x_{n}\right| \leqslant K$, then there is a sufficiently large $R$ such that

$$
\int_{B_{R}(0)}\left|u_{n}\right|^{m}>\bar{c}>0
$$

implying that $u \not \equiv 0$. On the other hand, we may have $\left|x_{n}\right| \rightarrow \infty$. In this case, let $v_{n}(x)=u_{n}\left(x+x_{n}\right)$. Hence, $v_{n} \rightarrow v$ and

$$
\int_{B_{1}(0)}\left|v_{n_{k}}\right|^{m}>\bar{c}>0
$$

thus, $v \not \equiv 0$. By (1.4), $v$ satisfies

$$
-\Delta_{m} v+|v|^{m-2} v=0 \text { in } \mathbb{R}^{N} \text { and } v \in W^{1, m}\left(\mathbb{R}^{N}\right),
$$

implying that $v \equiv 0$ in $\mathbb{R}^{N}$, which constitutes a contradiction.

Moreover, since $u \not \equiv 0$ by (2.1) and (2.4), we conclude that $u>0$ in $\mathbb{R}^{N}$.

By Lemma 2.5 below, the norm $\|u\|_{L^{\infty}\left(\mathbb{R}^{N}\right)}$ is small for sufficiently large $\lambda$. Thus, $u$ is indeed a positive solution of the original problem (1.1).

Remark 2.4. It is an open question as to whether $\tilde{\lambda}=\lambda^{*}$. In general $\tilde{\lambda}$ depends on $m$; for $m=2$ and $a \equiv 1$ the equality $\tilde{\lambda}=\lambda^{*}$ is valid (see $[\mathbf{1}]$ ).

Lemma 2.5. Let $u$ be a mountain pass solution of (1.1). Then

$$
\|u\|_{L^{\infty}\left(\mathbb{R}^{N}\right)} \leqslant C \lambda^{-1 /(p+1-m)}
$$

for some constant $C>0$ independent of $\lambda$ and $u$. 
Proof of Lemma 2.5. The critical value corresponding to a mountain pass solution $u$ is given by

$$
\begin{gathered}
c=\min _{\gamma \in \Gamma} \max _{t \in[0,1]} \hat{J}(\gamma(t))=\hat{J}(u), \\
\Gamma=\left\{\gamma \in C^{0}\left([0,1], W^{1, m}\left(\mathbb{R}^{N}\right)\right): \gamma(0)=0, \gamma(1)=e\right\},
\end{gathered}
$$

where $\hat{J}(0)=0$ and $\hat{J}(e) \leqslant 0$. In order to choose some value $e$, let $\varphi \in W^{1, m}\left(\mathbb{R}^{N}\right)$ with $\operatorname{supp}(\varphi) \subset\{a>0\}$ and $\varphi \geqslant 0$. Hence,

$$
\begin{aligned}
\hat{J}(t \varphi) & =t^{m}\|\varphi\|_{W^{1, m}\left(\mathbb{R}^{N}\right)}^{m}-\lambda \int_{\Omega} a(x) F(\lambda, t \varphi) \\
& \leqslant t^{m}\|\varphi\|_{W^{1, m}\left(\mathbb{R}^{N}\right)}^{m}-\frac{t^{p+1}}{p+1} \lambda\left(1-\varepsilon^{q-p}\right) \int_{\Omega} a(x) \varphi^{p+1} .
\end{aligned}
$$

We choose $t=t_{0}$, in such a way that $\hat{J}\left(t_{0} \varphi\right) \leqslant 0$, so that

$$
t_{0}^{p+1-m}=\frac{(p+1)\|\varphi\|_{W^{1, m}\left(\mathbb{R}^{N}\right)}^{m}}{\lambda\left(1-\varepsilon^{q-p}\right) \int_{\Omega} a(x) \varphi^{p+1}} .
$$

Let $e=t_{0} \varphi$. Then

$$
\|e\|_{W^{1, m}\left(\mathbb{R}^{N}\right)}=t_{0}\|\varphi\|_{W^{1, m}\left(\mathbb{R}^{N}\right)} \leqslant \frac{k}{\lambda^{1 /(p+1-m)}},
$$

where $k>0$ is a constant independent of $\lambda$. Hence,

$$
\begin{aligned}
c & \leqslant \max _{t>0} \hat{J}(t \varphi) \\
& \leqslant \max _{t>0}\left[t^{m}\|e\|_{W^{1, m}\left(\mathbb{R}^{N}\right)}^{m}-\frac{t^{p+1}}{p+1} \lambda\left(1-\varepsilon^{q-p}\right) \int_{\Omega} a(x)|e|^{p+1}\right] \\
& \leqslant t_{0}^{m}\|e\|_{W^{1, m}\left(\mathbb{R}^{N}\right)}^{m}-\frac{t_{0}^{p+1}}{p+1} \lambda\left(1-\varepsilon^{q-p}\right) \int_{\Omega} a(x)|e|^{p+1} \\
& \leqslant t_{0}^{m}\|e\|_{W^{1, m}\left(\mathbb{R}^{N}\right)}^{m} \leqslant\left(\frac{k_{1}}{\lambda^{1 /(p+1-m)}}\right)^{m},
\end{aligned}
$$

where $k_{1}$ is independent of $\lambda$.

Since $u$ is a solution,

$$
\left(\frac{1}{m}-\frac{1}{\theta}\right)\|u\|_{W^{1, m}\left(\mathbb{R}^{N}\right)}^{m} \leqslant \hat{J}(u)=c<\left(\frac{k_{1}}{\lambda^{1 /(p+1-m)}}\right)^{m}
$$

and

$$
\|u\|_{L^{m^{*}}\left(\mathbb{R}^{N}\right)} \leqslant k\|u\|_{W^{1, m}\left(\mathbb{R}^{N}\right)} \leqslant \frac{k_{1}}{\lambda^{1 /(p+1-m)}} .
$$

We are going to perform a Moser iterative scheme (see, for example, [6]). Here we need to keep track of the dependence on $\lambda$. 
Note that $m<p+1<m^{*}$ and, by (1.3) and (2.2),

$$
-\Delta_{m} u+u^{m-1} \leqslant \lambda u^{p} \quad \text { in } \mathbb{R}^{N} .
$$

For $M>0$ and $k>0$, define $v_{M}(y)=\inf \{u(y), M\}$ and $v(y)=\left(v_{M}(y)\right)^{k m+1}$. Using $v$ as a test function in (2.7), we obtain

$$
\begin{gathered}
(k m+1) \int v_{M}^{k m}\left|\nabla v_{M}\right|^{m}+\int v_{M}^{m+k m} \leqslant \lambda \int v_{M}^{p+k m+1}, \\
\frac{k m+1}{(k+1)^{m}} \int\left|\nabla\left(v_{M}\right)^{k+1}\right|^{m} \leqslant \lambda \int v_{M}^{(k+1) m+p+1-m}, \\
\frac{1}{c_{1}} \frac{k m+1}{(k+1)^{m}}\left(\int\left|\left(v_{M}\right)^{(k+1) m^{*}}\right|\right)^{m / m^{*}} \leqslant \lambda\left(\int v_{M}^{(k+1) m l}\right)^{1 / l}\left(\int v_{M}^{m^{*}}\right)^{(p+1-m) / m^{*}} .
\end{gathered}
$$

From now on $c_{i}, i=1,2,3,4$, denote positive constants independent of $\lambda$. Note that

$$
l=\frac{m^{*}}{m^{*}-(p+1-m)}
$$

and observe that $m<p+1<m^{*}$, so that $0<p+1-m<m^{*}-m$. Then $m<$ $m^{*}-(p+1-m)<m^{*}$. From now on the norm spaces correspond to functions defined in $\mathbb{R}^{N}$. Thus,

$$
\begin{aligned}
& \left\|v_{M}\right\|_{L^{m^{*}(k+1)}} \\
& \qquad c_{2}^{1 /(k+1)}\left(\frac{k+1}{(k m+1)^{1 / m}}\right)^{1 /(k+1)} \lambda^{1 /((1+k) m)}\left\|v_{M}\right\|_{L^{(k+1) m l}}\left\|v_{M}\right\|_{L^{m^{*}}}^{(p+1-m) /(m(k+1))} .
\end{aligned}
$$

Letting $M \rightarrow \infty$ yields

$$
\|u\|_{L^{m^{*}(k+1)}} \leqslant\left(c_{2} \lambda^{1 / m}\|u\|_{L^{m^{*}}}^{(p+1-m) / m}\right)^{1 /(k+1)}\|u\|_{L^{(k+1) m l}}\left(\frac{k+1}{(k m+1)^{1 / m}}\right)^{1 /(k+1)} .
$$

Define $k_{1}$ as $\left(k_{1}+1\right) m l=m^{*}$. Note that $k_{1}+1=m^{*} / m l>1$ and therefore

$$
\|u\|_{L^{m^{*}\left(k_{1}+1\right)}} \leqslant\left(c_{2} \lambda^{1 / m}\|u\|_{L^{m^{*}}}^{(p+1-m) / m}\right)^{1 /\left(k_{1}+1\right)}\|u\|_{L^{m^{*}}}\left(\frac{k+1}{(k m+1)^{1 / m}}\right)^{1 /\left(k_{1}+1\right)} .
$$

By induction, we define $\left(k_{n}+1\right) m l=m^{*}\left(k_{n-1}+1\right)$. Then $k_{n}+1=\left(m^{*} / m l\right)^{n}$ and

$$
\begin{aligned}
& \|u\|_{L^{m^{*}\left(k_{n}+1\right)}} \\
& \leqslant\left(c_{2} \lambda^{1 / m}\|u\|_{L^{m^{*}}}^{(p+1-m) / m}\right)^{\sum_{j=1}^{n} 1 /\left(k_{j}+1\right)} \prod_{j=1}^{n}\left(\left(\frac{k_{j}+1}{\left(k_{j} m+1\right)^{1 / m}}\right)^{1 / \sqrt{k_{j}+1}}\right)^{1 / \sqrt{k_{j}+1}}\|u\|_{L^{m^{*}}}
\end{aligned}
$$

and hence

$$
\|u\|_{L^{m^{*}\left(k_{n}+1\right)}} \leqslant\left(c_{2} \lambda^{1 / m}\|u\|_{L^{m^{*}}}^{(p+1-m) / m}\right)^{\sum_{j=1}^{n} 1 /\left(k_{j}+1\right)} c_{3}^{\sum_{j=1}^{n} 1 / \sqrt{k_{j}+1}}\|u\|_{L^{m^{*}}} .
$$


Since

$$
\sum_{j=1}^{\infty} \frac{1}{k_{j}+1}=\sum_{j=1}^{\infty}\left(\frac{m l}{m^{*}}\right)^{j}=\frac{1}{1-(m l / m *)}-1=\frac{m^{*}}{m^{*}-m l}-1=\frac{m}{m^{*}-(p+1)},
$$

we obtain

$$
\begin{aligned}
\|u\|_{L^{\infty}} & \leqslant c_{4} \lambda^{1 /\left(m^{*}-(p+1)\right)}\|u\|_{L^{m^{*}}}^{(p+1-m) /\left(m^{*}-(p+1)\right)+1} \\
& =c_{4} \lambda^{1 /\left(m^{*}-(p+1)\right)}\|u\|_{L^{m^{*}}}^{\left(m^{*}-m\right) /\left(m^{*}-(p+1)\right)}
\end{aligned}
$$

By (2.6), we obtain the desired estimate.

Acknowledgements. The authors have been supported by the UTA Chilean grant no. 4732-04 and the Brazilian scientific organizations CNPq grant no. 478896/2003-4 and FAPESP grant no. 04/06678-0.

\section{References}

1. H. BerESTYCKi AND P.-L. Lions, Existence of a ground state in nonlinear equations of the Klein-Gordon type, in Variational Inequalities and Complementarity Problems, Proc. Int. School of Nuclear Physics, Erice, 1978, pp. 35-51 (Wiley, 1980).

2. H. Berestycki and P.-L. Lions, Nonlinear scalar field equations, I, Existence of a ground state, Arch. Ration. Mech. Analysis 82 (1983), 313-345.

3. A. Chaludu-Simon and P. Volkmann, Existence of ground states with exponential decay for semilinear elliptic equations in $R^{n}$, J. Diff. Eqns 76 (1988), 374-390.

4. W. Y. DING AND W. M. NI, On the existence of positive entire solutions of a semilinear elliptic equation, Arch. Ration. Mech. Analysis 91 (1986), 283-308.

5. P.-L. LiONS, The concentration-compactness principle in the calculus of variations, the limit case, I, Rev. Mat. Iber. 1 (1985), 145-201.

6. M. OTANI, Existence and nonexistence of nontrivial solutions of some nonlinear degenerate elliptic equations, J. Funct. Analysis 76 (1988), 140-159.

7. W. Strauss, Existence of solitary waves in higher dimensions, Commun. Math. Phys. 55 (1977), 149-162.

8. X. P. ZHU, Multiple entire solutions of a semilinear elliptic equation, Nonlin. Analysis 12 (1988), 1297-1316. 